\title{
Platelet proteome and clopidogrel response in patients with stable angina undergoing percutaneous coronary intervention
}

\author{
Elisabetta Volpi a, Laura Giusti ${ }^{\text {b }}$, Federica Ciregia ${ }^{\text {b }}$, Ylenia Da Valle ${ }^{\text {b }}$, Gino Giannaccini ${ }^{\text {b }}$, Sergio Berti ${ }^{\text {, }}$ \\ Aldo Clerico ${ }^{a}$, Antonio Lucacchini ${ }^{\mathrm{b}, *}$ \\ a Scuola Superiore Sant'Anna, Pisa, Italy \\ ${ }^{\mathrm{b}}$ Department of Psychiatry, Neurobiology, Pharmacology and Biotechnology, University of Pisa, Pisa, Italy \\ c Department of Cardiology, Heart Hospital, Gabriele Monasterio Foundation, Massa, Italy
}

\section{A R T I C L E I N F O}

\section{Article history:}

Received 5 September 2011

Received in revised form 27 January 2012

Accepted 23 March 2012

Available online 3 April 2012

\section{Keywords:}

Proteomics

Platelets

Clopidogrel

Percutaneous coronary intervention

Antiplatelet therapy

\section{A B S T R A C T}

Objectives: We analyzed the platelet proteome of circulating platelets during the onset of clopidogrel therapy in patients with stable angina underwent percutaneous coronary intervention in order to investigate the mechanisms that control platelet reactivity and clopidogrel response in this context.

Design \& methods: Twenty patients were enrolled in this study. Blood samples were collected before coronary angiography (T0), $12 \mathrm{~h}$ after $600 \mathrm{mg}$ of clopidogrel (T1) and $24 \mathrm{~h}$ after percutaneous coronary intervention (PCI) (T2). Platelet reactivity, Clopidogrel response and proteomic analysis were examined.

Results: Clopidogrel loading dose produced a significant inhibition in all markers of platelet activation in both flow cytometry and aggregation tests. Among the proteins found differentially expressed, eighteen were identified by MS/MS analysis and they resulted involved in the cytoskeleton rearrangement (profilin-1, calpain, $\alpha$-soluble NSF attachment protein, thrombospondin), in the energetic metabolism (ubiquitin-like modifier-activating enzyme 1 , protein-L-isoaspartate-(D-aspartate) 0 -methyltransferase and nucleoside diphosphate kinase B) and in the oxidative stress (heat shock $70 \mathrm{kDa}$ protein 5 and anti-stress induced phosphoprotein 1.

Conclusions: The present study provides novel information on platelet proteome changes associated with platelet activation and clopidogrel response. This investigation supports the development of further proteomic studies for the identification of novel platelet biomarkers.

(C) 2012 The Canadian Society of Clinical Chemists. Published by Elsevier Inc. All rights reserved.

\section{Introduction}

Platelets are anucleate cells that play a primary role in physiologic hemostasis and repair injury to the vasculature. They circulate in an

Abbreviations: PCI, percutaneous coronary intervention; 2DE, two-dimensional gel electrophoresis; NSTE-ACS, non-ST segment elevation acute coronary syndrome; NSAID, non-steroidal anti-inflammatory drugs; COX-2, cyclooxygenase 2; PRP, Platelet-rich plasma; FITC, fluorescein isothiocyanate; PE, phycoerythrin; RT, room temperature; PBS, phosphate buffered-saline; RFI, relative fluorescence intensity: VASP, Vasodilator-stimulated phosphoprotein; PRI, Platelet Reactivity Index; LTA, Light Transmission Aggregometry; CHAPS, 3-[(3-Cholamidopropyl)dimethylammonio]-1propanesulfonate; DTT, dithiothreitol; IEF, Isoelectrofocusing; IAA, Iodoacetamide; SDS, sodium dodecyl sulfate; TEMED, tetramethylethylenediamine; IPGs, Immobiline Dry-Strips; ECL, enhanced chemiluminescence; $\alpha$-SNAP, Anti $\alpha$-soluble NSF attachment protein; STIP1, anti-stress induced phosphoprotein 1; CAD, coronary artery disease; WB, Western blot; THBS-1, thrombospondin.

* Corresponding author at: Department of Psychiatry, Neurobiology, Pharmacology and Biotechnology, Via Bonanno 6, 56126 Pisa, Italy. Fax: + 390502219609.

E-mail addresses: volpi@ifc.cnr.it (E. Volpi), giusti@farm.unipi.it (L. Giusti), ciregia@farm.unipi.it (F. Ciregia), ylenia.davalle@gmail.com (Y. Da Valle), ggino@farm.unipi.it (G. Giannaccini), sergio.berti@ftgm.it (S. Berti), clerico@ifc.cnr.it (A. Clerico), lucas@farm.unipi.it (A. Lucacchini). unactivated state, but in response to vascular injury platelets adhere to the sub-endothelium, where their aggregation leads progressively to the thrombus formation, restraining the hemorrhage.

Following vascular injury, as the endothelial damage caused by coronary stenting, the plaque's highly prothrombotic contents is exposed to the blood flow, resulting in the adherence of platelets to the damaged area, the release of activating agonists, stored in platelet intracellular granules, that amplify the stimulation to the adjacent platelets and the generation of a platelet-rich thrombus.

In order to prevent this acute manifestation, the use of antiplatelet drugs showed clear benefits over time, validated by several studies and clinical trials [1]. The current guidelines, in fact, recommend the oral administration of a dual antiplatelet therapy, with an ADP-receptor antagonist like clopidogrel in association with aspirin [1]. Clopidogrel is widely used as platelet inhibitor for coronary angioplasty procedures especially in the setting of coronary stent implantation [2].

It is clear that undergoing to percutaneous coronary intervention (PCI) with an adequate platelet inhibition level is associated with a lower incidence of peri- and post-procedural adverse ischemic complications compared to inadequate pre-treatment. Therefore a loading dose of $600 \mathrm{mg}$ of clopidogrel is now recommended as soon 
as possible before an urgent PCI and at least $6 \mathrm{~h}$ before an elective procedure [1]. The high dose pre-treatment is generally associated with an adequate and approximately homogeneous antiplatelet effect $[3,4]$, but the variability in platelet response and the mechanisms connected with this phenomenon are still unclear. Traditional platelet aggregation tests and the analysis of the platelet surface markers using flow cytometry continue to serve as remarkable tests for platelet function studies [5]. Beside them, the use of proteomics holds great promise for better understanding platelet biology and identifying potential diagnostic markers and therapeutic targets.

The platelet proteome is altered in patients with coronary artery diseases possibly because of the ongoing atherosclerotic process [6]. Recently, a high-resolution two-dimensional gel electrophoresis (2DE)-based proteome analysis of platelets from non-ST segment elevation acute coronary syndrome (NSTE-ACS) patients has been performed, showing that proteomics can be used to follow platelet proteome changes during the follow-up [7]. The administration of antiplatelet drugs and the stent placement are responsible for multiple stimuli that may rapidly modify the expression of plateletrelated proteins. So we study the platelet proteome of patients underwent clopidogrel treatment and elective $\mathrm{PCI}$ in order to investigate the mechanisms that control platelet reactivity in this context.

\section{Materials and methods}

\section{Patients selection}

Twenty patients with stable coronary artery disease were enrolled in this study. The protocol was approved by the local ethical committee and the informed consent was obtained from each subject before the enrollment. All patients had received daily aspirin therapy of $100 \mathrm{mg}$

A
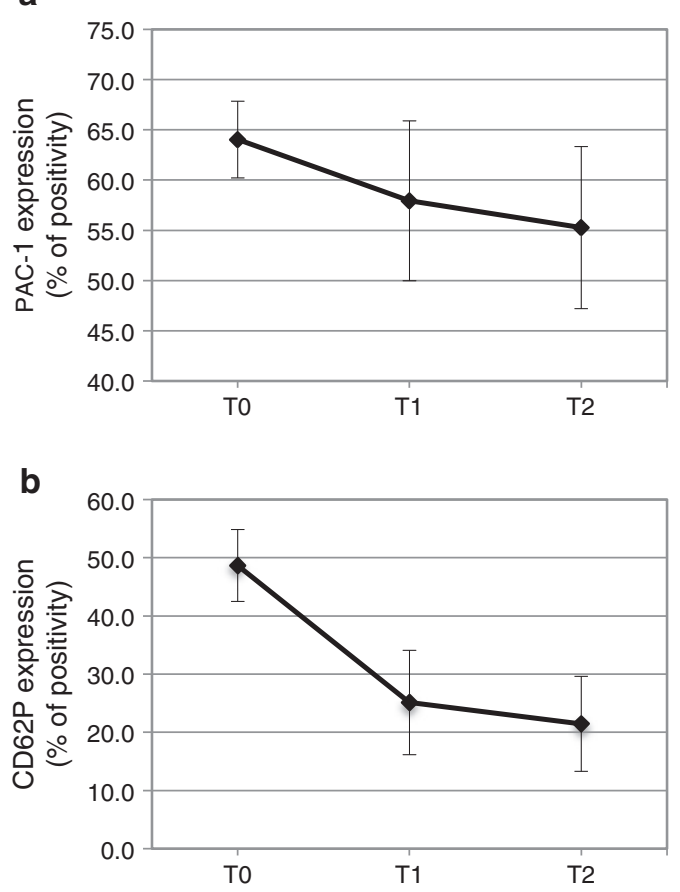

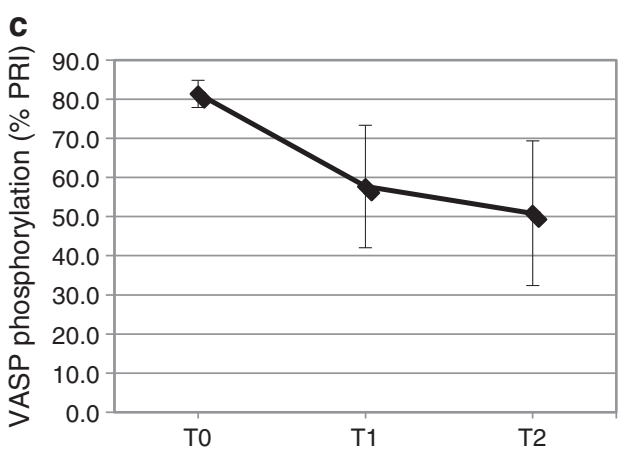

B

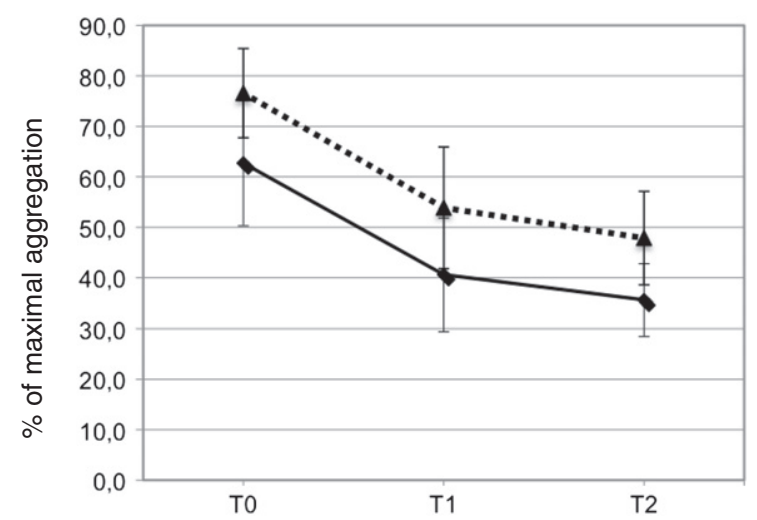

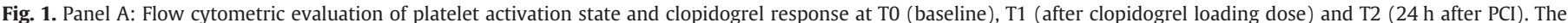

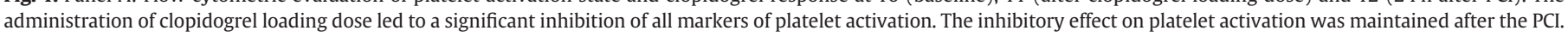

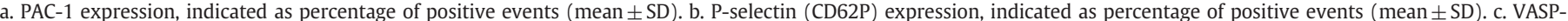

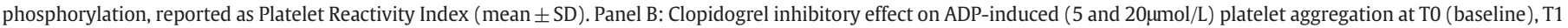

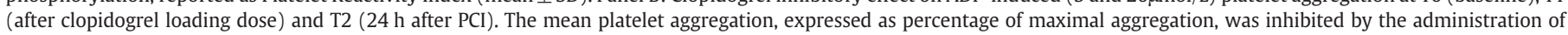
clopidogrel loading dose and the effect was protracted after the PCI. 
for at least 1 month before the study. Exclusion criteria were acute coronary syndrome or revascularization within the last 6 months; concurrent ingestion of non-steroidal anti-inflammatory drugs (NSAID, including COX-2 selective anti-inflammatory drugs), clopidogrel, ticlopidine, dipyridamole or warfarin; platelet count outside the 100 to $450 \times 10^{9} / \mathrm{L}$ range; hematocrit $<25 \%$ or haemoglobin $<100 \mathrm{~g} / \mathrm{L}$; and chronic renal failure.

All patients were admitted in the Cardiology Department of the Heart Hospital in Massa for symptomatic stable angina and investigated with a diagnostic angiography to confirm the necessity of coronary revascularization. They received a $600 \mathrm{mg}$ clopidogrel loading dose $\geq 12 \mathrm{~h}$ before the PCI. After the procedure all patients started the maintenance therapy with clopidogrel $75 \mathrm{mg}$ /day for at least 6 months and continued aspirin $100 \mathrm{mg} /$ day indefinitely.

\section{Samples collection}

Blood samples were drawn from an antecubital vein using a 19-gauge needle and the first $2.5 \mathrm{~mL}$ were discarded. All samples were collected in Vacutainer tubes (Becton Dickinson, San Jose, CA, http://www.bdbiosciences.com) containing 3.8\% sodium citrate as anticoagulant. Patients' blood was drawn at three different time points: before the PCI (T0), $12 \mathrm{~h}$ after the loading dose of clopidogrel (T1) and $24 \mathrm{~h}$ after stenting (T2). Platelet-rich plasma (PRP) was isolated by slow centrifugation at $22{ }^{\circ} \mathrm{C}$ at $100 \mathrm{~g}$ for $15 \mathrm{~min}$. The platelet pellet was then isolated by an additional centrifugation at $1000 \mathrm{~g}$ for $20 \mathrm{~min}$ and stored at $-80^{\circ} \mathrm{C}$ until further protein extraction.

\section{Platelet activation markers: PAC-1 and CD62P}

The determination of the platelet surface expression of $\alpha$ IIb $\beta 3$ integrin and P-selectin was performed by flow cytometry. Whole blood samples were diluted 1:6 in HEPES phosphate buffer (N-2hydroxy-ethyl-piperazine- $\mathrm{N}^{\prime}$-2-ethanesulfonic acid), containing fluorescein isothiocyanate (FITC) PAC-1 (Becton Dickinson, San Jose, CA, http://www.bdbiosciences.com) or phycoerythrin (PE) conjugated CD62P (Pharmingen, San Diego, CA, http://www.pharmingen.com) monoclonal antibodies. Platelets were identified with the double labeling method, using CD41a (FITC; Becton Dickinson, San Jose, CA) or CD61 (PE; Pharmingen, San Diego, CA). Platelet activation was induced with $5 \mu \mathrm{mol} / \mathrm{L}$ ADP (Sigma Chemical, St. Louis, MO, http:// www.sigmaaldrich.com). The unactivated (resting) samples were used to establish the baseline expression of PAC- 1 and CD62P on the platelet surface. Each test was performed in parallel with the mouse isotype control. All samples were incubated for $8 \mathrm{~min}$ at room temperature (RT) in the dark and subsequently fixed and diluted $1: 10$ with $1.0 \%$ ice-cold paraformaldehyde solution in PBS for analysis by flow cytometry (FACScan; Becton Dickinson, San Jose, CA, http:// www.bdbiosciences.com). PAC-1 and P-selectin expression levels were calculated as percentage of positive events or relative fluorescence intensity (RFI), expressed as linear fluorescence channels subtracted by the median value of the isotype-matched control histogram.

\section{Clopidogrel response}

The platelet response to clopidogrel was evaluated through the analysis of intraplatelet phosphorylation level of the Vasodilatorstimulated phosphoprotein (VASP assay) by flow cytometry and with an aggregation test using optical aggregometry. The intraplatelet VASP phosphorylation level reflects the P2Y12 receptor antagonism, thus clopidogrel efficacy. VASP phosporylation was measured by whole blood flow cytometry using PLT VASP/P2Y12 kit (Biocytex, Marseille, France, http://www.biocytex.fr). The staining procedure and the calculation of the platelet reactivity index (PRI) were performed according to the manufacturer's instructions.

Light transmittance aggregometry (LTA) has been the most widely used technique to monitor the effects of antiplatelet agents. Platelet aggregation was induced by adding ADP 5 and $20 \mu \mathrm{mol} / \mathrm{L}$ to $475 \mu \mathrm{L}$ of $\mathrm{PRP}$ in an aggregometer (Chrono-log Aggregometer, series 490; Harveton, PA). Platelet aggregation was expressed as the maximum percentage change in light transmittance, with platelet-poor plasma used as a reference for $100 \%$ aggregation.

\section{Protein extracts preparation}

Pellets of platelets were resuspended in $350 \mu \mathrm{l}$ of rehydration solution ( $7 \mathrm{~mol} / \mathrm{L}$ Urea, $2 \mathrm{~mol} / \mathrm{L}$ thiourea, 4\% 3-[(3-Cholamidopropyl) dimethylammonio]-1-propanesulfonate (CHAPS), $60 \mathrm{mmol} / \mathrm{L}$ dithiothreitol (DTT), $0.002 \%$ bromophenol blue), sonicated 4 times for $5 \mathrm{~s}$ and incubated for $30 \mathrm{~min}$ at RT. After incubation the samples were centrifuged for $10 \mathrm{~min}$ at $15,000 \mathrm{~g}$ to remove undissolved material. Protein concentrations were measured with a RC-DC Protein Assay from Bio-Rad, using bovine serum albumin as standard. The mean value \pm SD of $\mu$ g of proteins obtained for T0, T1 and T2 samples were $1502 \pm 353,1563 \pm 416$ and $1493 \pm 359$ respectively.

\section{DE analysis}

Isoelectrofocusing (IEF) was carried out by using $18 \mathrm{~cm}$ IPG-strips (Immobiline Dry-Strips, GE Healthcare, UK, http://www.gehealthcare. com) with a non-linear, pH 3-10, gradient. For analytical gels $150 \mu \mathrm{g}$ proteins were filled up to $350 \mu \mathrm{l}$ with rehydration buffer supplemented with $1 \%(\mathrm{v} / \mathrm{v})$ pharmalytes, $\mathrm{pH} 3-10$. 2DE was performed using the Immobiline-polyacrylamide system as previously described [8]. For preparative gels $1500 \mu \mathrm{g}$ of proteins pooled from equal amounts of samples were used. The analytical gels for all platelet protein samples were processed in duplicate.

\section{Staining and image analysis}

Analytical gels were stained with ammoniacal silver nitrate as previously described [9]. Preparative gels for mass spectrometric analysis were stained with Coomassie Brilliant Blue G-colloidal [10].

Table 1

Patients demographics, medical history and concomitant medication.

\begin{tabular}{ll}
\hline Demographics & \\
\hline Age (yr) & $68.4 \pm 7.7$ \\
Male sex (\%) & 75 \\
BMI $\left(\mathrm{kg} / \mathrm{m}^{2}\right)$ & $28.8 \pm 3.5$ \\
Medical history (\%) & \\
Hypertension & 80 \\
Hyperlipidemia & 95 \\
Diabetes mellitus & 10 \\
Smoking (current) & 2 \\
Family history of CAD & 45 \\
Concomitant medications (\%) & \\
ACE inhibitors & 65 \\
Sartans & 25 \\
$\beta$-blockers & 70 \\
Diuretics & 35 \\
Calcium channel blockers & 25 \\
Proton pump inhibitors & 85 \\
Statins & 88 \\
Target lesion coronary artery (\%) & \\
Left anterior descending & 75 \\
Left circumflex & 20 \\
Right & 40 \\
Stent implantation (\%) & \\
DES & 80 \\
BMS & 20 \\
[Data were expressed as mean \pm SD or \%] & \\
\hline
\end{tabular}


The stained gels were scanned with an Epson Expression 1680 Pro scanner and the images were analyzed with the Image-Master 2D Platinum 6.01 (GE Healthcare, UK, http://www.gehealthcare.com) software program. For the analysis the samples were grouped in three different classes corresponding to T0, T1 and T2 time points. The proteins with a $\geq 1.5$-fold spot quantity change in each class were selected and identified by MALDI TOF-TOF mass spectrometry.

Maldi TOF-TOF acquisition, database searching and criteria for protein identification

Spots of interest were cut out from the master gel, treated and analyzed as previously described [11].Scaffold (version Scaffold_3_00_03, Proteome Software Inc., Portland, OR) was used to validate MS/MS based peptide and protein identifications. Peptide identifications were accepted if they could be established at greater than $95.0 \%$ probability as specified by the Peptide Prophet algorithm [12]. Protein identifications were accepted if they could be established at greater than $95.0 \%$ probability and contained at least 2 identified peptides. Protein probabilities were assigned by the Protein Prophet algorithm [13]. Proteins composed of similar peptides and that could not be differentiated based on MS/MS analysis alone were grouped to satisfy the principles of parsimony.

\section{Western blot analysis}

Alpha-soluble NSF attachment protein ( $\alpha$-SNAP), Stress induced phosphoprotein 1 (STIP1) and Profilin 1 were assessed by 1-D Western blot (WB) analysis.

Amounts of the samples corresponding to $20 \mu \mathrm{g}$ of proteins for $\alpha$ SNAP, $3 \mu \mathrm{g}$ for STIP1, and $2.5 \mu \mathrm{g}$ for Profilin-1 were mixed with Laemmli solution and heated $5 \mathrm{~min}$ at $100{ }^{\circ} \mathrm{C}$. The samples were then loaded on $12 \%$ polyacrylamide gels, electrotransferred to nitrocellulose membranes and incubated with primary antibodies [11]. We used as primary antibodies: rabbit polyclonal $\alpha$-SNAP antibody (1:500), rabbit polyclonal STIP1 (1:500) and rabbit polyclonal Profilin-1 (1:1000). For secondary antibody a goat anti-rabbit, 1:10,000 dilution, was used. For a negative control, the primary antibody was omitted. Proteins were revealed with ECL detection method. For each tested antibodies WB analysis was performed on all platelets samples in duplicate. All primary antibodies were from Abcam plc, UK, (http://abcam.com), secondary antibody was from
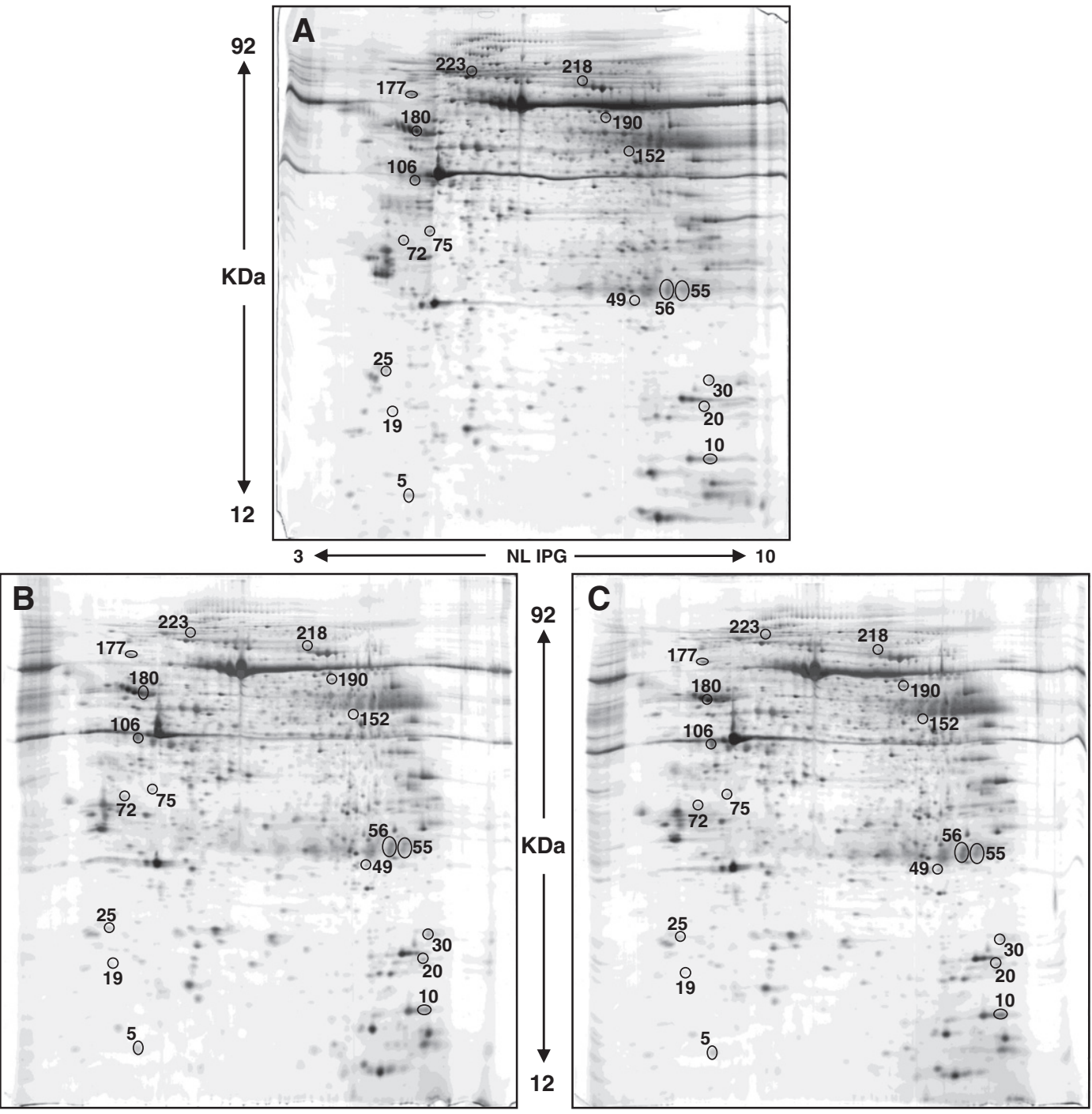

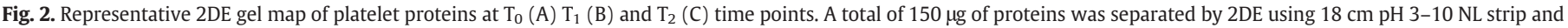

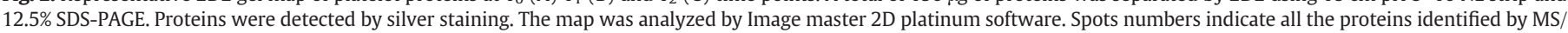
MS and refer to the number reported in Table 2. 
Stressgen, MI (www.enzolifesciences.com). To visualize the different expression of various products of profilin-1, aliquots of $300 \mu \mathrm{g}$ of proteins were separated by 2DE using 3-10 non-linear strips $18 \mathrm{~cm}$ before western blot analysis with specific antibody. For this experiment the platelet samples appertaining to the same time point (T0, T1 and T2), were pooled and processed in triplicate.

\section{Statistical analysis}

Data for continuous variables as the results of the platelet function study were expressed as mean \pm standard deviation. Patients' differences in platelet function at various time points (Figs. 1A and B) were analyzed by the post hoc Scheffe's test after paired data ANOVA. The optical density of the proteins was expressed as percentage of the volume (mean $\pm S D$ ). The optical density of the proteins was expressed as percentage of the volume (mean $\pm S D$ ). The significance of the differences for the proteomic study was calculated using repeated measures Anova tests. $p<0.05$ were considered to indicate statistical significance.

\section{Results}

\section{Patient's characteristics}

A total of 20 consecutive patients who fulfilled the enrollment criteria were prospectively included. Demographic, clinical and angiographic characteristics of the studied population are summarized in Table 1.

\section{Platelet function study}

On average, the administration of the clopidogrel loading dose produced a significant inhibition in all markers of platelet activation, as demonstrated by the significant differences observed between T0 and T1 experimental time points, in both flow cytometry tests [PAC-1 $64.0 \pm 3.8$ vs $57.9 \pm 7.9 p=0.0006 ;$ CD62P $48.7 \pm 6.2$ vs $25.1 \pm 8.9$ $p<0.0001$; PRI $81.4 \pm 3.5$ vs $57.7 \pm 20.7 p<0.0001]$ and aggregation tests [ADP $5 \mu \mathrm{mol} / \mathrm{L} \quad 62.7 \pm 12.4$ vs $40.6 \pm 11.2 p<0.0001$; ADP $20 \mu \mathrm{mol} / \mathrm{L} 76.7 \pm 8.8$ vs $53.9 \pm 12.0 p<0.0001]$, as reported in Figs. $1 \mathrm{~A}$ and $\mathrm{B}$.

The treatment with proton pump inhibitors (PPI) did not influence the clopidogrel response in our population, in fact there was no significant difference in platelet function results between patients treated or not with PPI ( $p=n s)$.

In the samples collected after the PCI (T2) we observed that the mean values of each platelet function test were comparable to those obtained at T1 ( $p=$ ns, data not shown). These data indicate that the revascularization procedure did not generate a re-activating response, suggesting the efficacy of the antiplatelet therapy in all patients.

2DE platelet proteome and mass-spectrometry identification of differentially expressed proteins

Platelet protein extraction resulted in reproducible highresolution 2D gels. Silver staining of 2D gels grouped as time points, showed similar protein patterns within a non-linear $\mathrm{pH}$ range from 3 to 10 with matching values over $80 \%$. Typical $2 \mathrm{D}$ gel images of platelet protein extracts from T0, T1 and T2 are shown in Figs. 2A, B, and C, respectively. By computational 2D gel image comparison, a total of 24 protein spots were found to be differentially expressed, each exhibiting $\geq 1.5$ fold-change (either increase or decrease) of mean value spot intensity (\% of volume) among the three different time points. Differentially expressed protein spots were subsequently subjected to MS/MS analysis. Of the 24 spots, $75 \%$ were identified by MS/MS spectrometry. A list of the eighteen identified proteins,

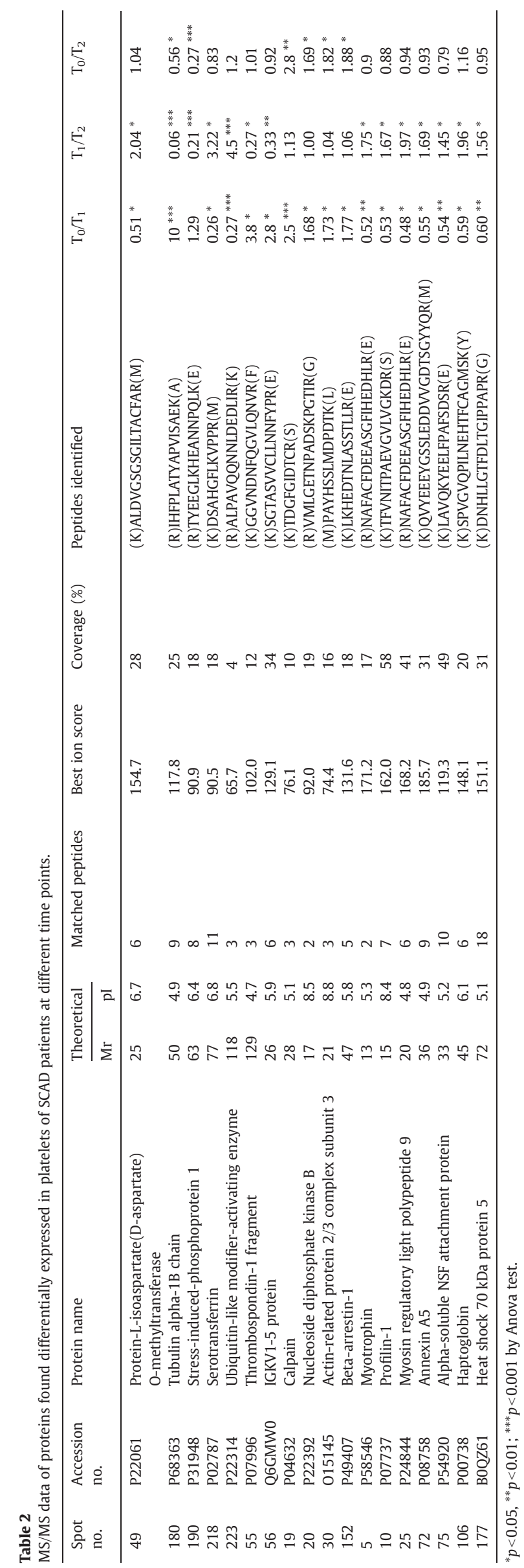


MW, pI, score and coverage values of MS/MS ratio of change in the expression level and $p$-values is shown in Table 2. In Fig. 3 the selection of differentially expressed proteins is depicted on enlarged gel images. Proteins identified by 2D/MS/MS proteomics were classified into functional categories and were associated with cytoskeleton, energetic metabolism, oxidative stress and inflammatory response.

\section{Validation of SNAP, STIP and Profilin-1 by western blotting analysis}

Western blot analysis with specific antibodies was used to validate the expression changes of SNAP, STIP-1 and Profilin-1 in platelet samples at all three time points. All platelet samples were tested to evaluate whether the observed protein expression changes were confirmed. A representative immunoblot for each protein tested is shown in Fig. 4. For each tested protein the optical density of specific immunoreactive band was determined and the resulting mean values $\pm \mathrm{SD}$ were compared (T0, T1, T2) (Fig. 4, bar graphs). For profilin-1, we could not confirm with 1D western blot the difference of expression found by 2DE analysis (data not shown). So a 2DE blot was performed and Fig. 4 shows the immunoblot of profilin- 1 obtained after reaction with specific antibody demonstrating a different pattern in the selected time points. The immunoreactive spots corresponded to pI values ranging from 6.9 (spot 1) to 9 (spot 6). After statistical analysis, except for spot 3 (pI 7.95), a significant increase for all other spots was detected at T1 when compared both with T0 and T2 conditions. The computation of theoretical pI value on the complete sequence of profilin-1 resulted 8.47 , as calculated by UniProtKB/SwissProt service. This value corresponded to $\mathrm{pI}$ value of spot 5 for which we detected an increase of expression of 2.5 fold after clopidogrel administration, that then disappeared after PCI.

\section{Discussion}

In the last decade the development and application of proteomics have offered the opportunity to investigate the proteins involved in the pathophysiology of several diseases. Recent studies have analyzed the platelet proteome in resting condition, after various stimuli or as subfractions (i.e. lysate, secretome) [14]. Furthermore platelets have been studied in healthy volunteers and in patients affected by coronary artery diseases [15], but the evaluation using a proteomic approach of the platelet response during the onset of antiplatelet therapy and the $\mathrm{PCI}$ is still unknown.

We analyzed the platelet proteome of 20 patients with stable angina, who underwent elective $\mathrm{PCI}$ and stent placement. The results from aggregation tests, the analysis of the platelet surface markers with flow cytometry and clopidogrel response were similar among those patients. The identified protein changes, connected to the onset of the clopidogrel therapy and the revascularization, showed an increase in the energy metabolism enzymes and alterations in the proteins associated with cytoskeleton-based processes, both of which indicate platelet activation.

The activation of platelets by an agonist stimulates different signal-transduction pathways that are initially mediated through an increase in the intracellular calcium. The calcium-dependent events include changes in the internal organization, the cytoskeleton rearrangement, the exocytosis of granule contents, the production of thromboxane $\mathrm{A}_{2}$ and finally the activation of

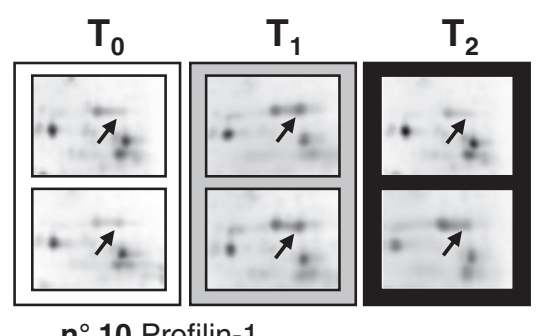

$n^{\circ} 10$ Profilin-1
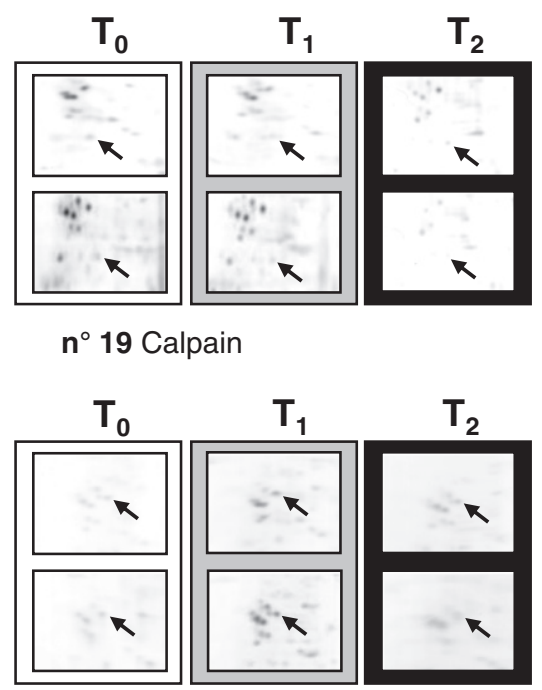

$n^{\circ} 25$ Myosin 9

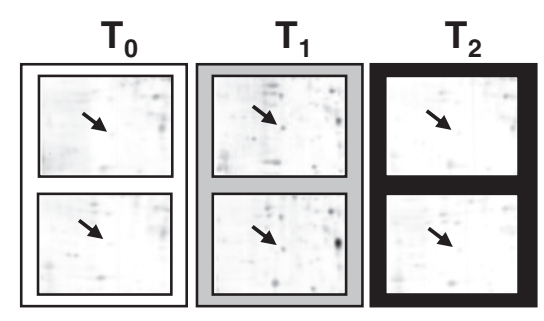

$n^{\circ} 75$ SNAP
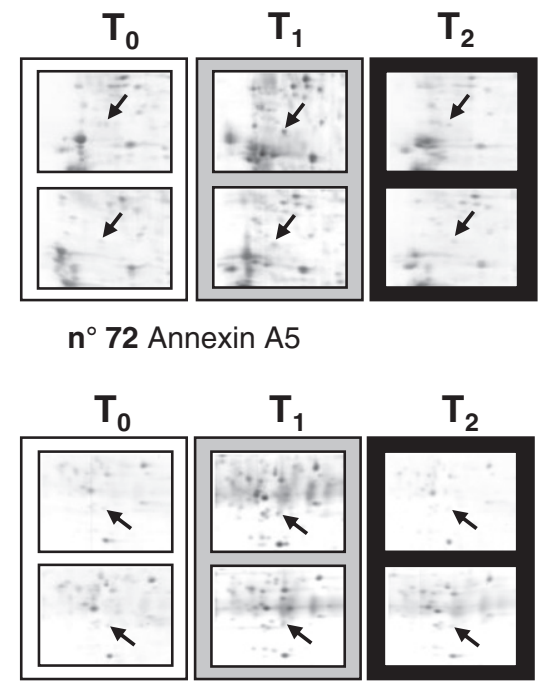

$n^{\circ} 49$ Protein-L-isoaspartate(Daspartate) O-methyltransferase

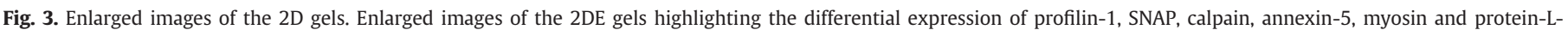
isoaspartate(D-aspartate) O-methyltransferase for two representative gels for each time. The spots of interest are indicated by the arrows. 
190 STIP-1
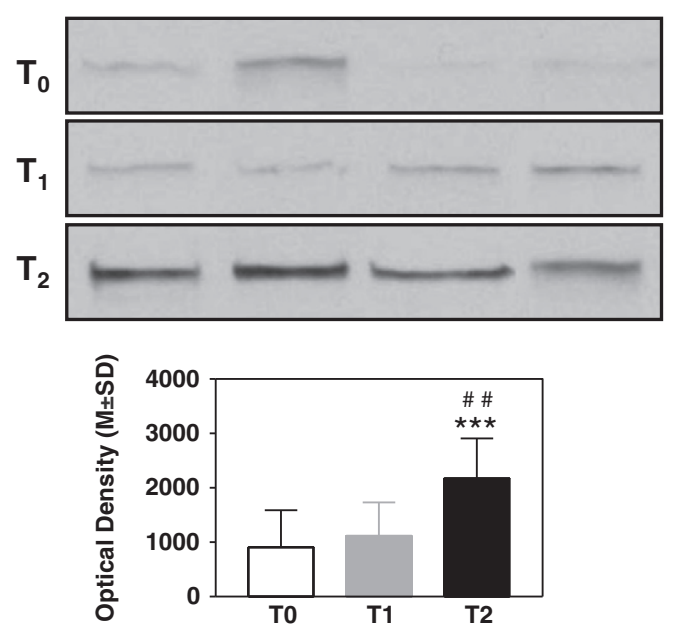

75 SNAP
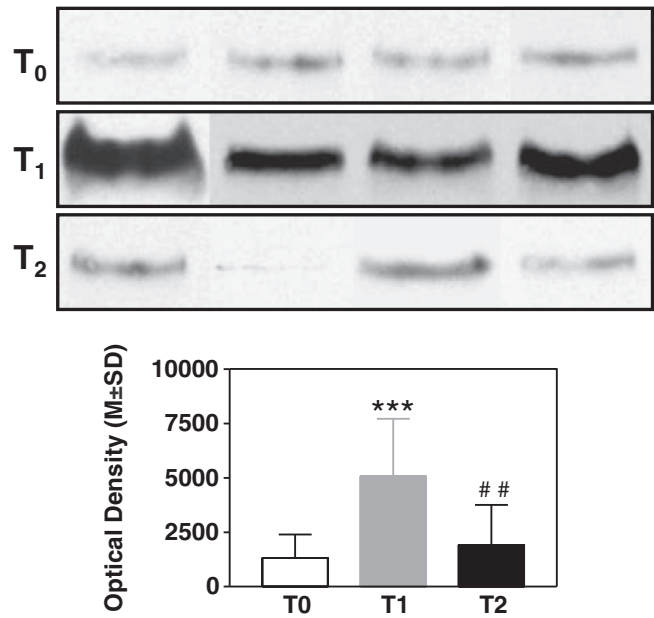

\section{PROFILIN-1}

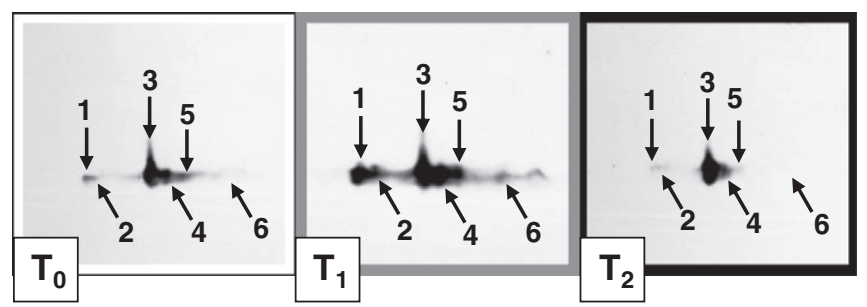

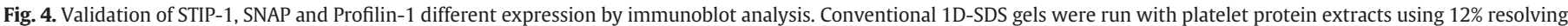

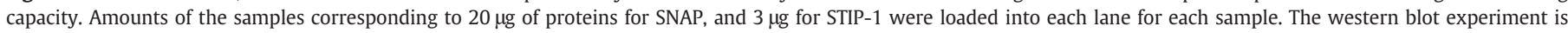

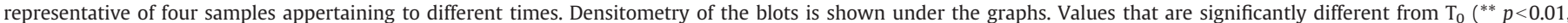

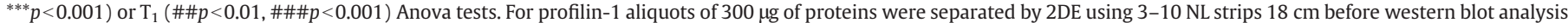
with specific antibody. The image is representative of experiments performed in triplicate.

fibrinogen receptors on the platelet surface. In platelets, mediators of the calcium signal include protein kinase $C$, calpain, calmodulin, phospholipase $\mathrm{A}_{2}$ and the annexins.

In this context, we interestingly observed that calpain, an intracellular calcium dependent cysteine proteinase, was found only at T0, before the antiplatelet treatment and the PCI. Calpain has been localized in the cytoskeletal fraction of thrombin-stimulated platelets and in points of attachment between cells and the extracellular matrix [16]. Activation of calpain leads to a remodeling of the platelet cytoskeleton and regulates the association of the cytoskeleton with plasma membrane glycoproteins. In particular, the formation of integrin-cytoskeletal signaling complexes is thought to be critical for the phosphorylation of multiple cytoskeletal proteins and for the stable incorporation of integrin $\alpha \operatorname{IIb} \beta 3$ into the contractile cytoskeleton [17]. Thus the elevated expression of calpain only at T0 supports the evidence of a pathologic condition of platelet activation in patients with CAD. This hyper-activation becomes successfully controlled by the clopidogrel loading dose and after PCI.

Furthermore the different expression of other proteins like THBS1 fragment, $\alpha$-SNAP and profilin- 1 may describe other mechanisms involved in platelet reactivity in this context. THBS-1 is an adhesive glycoprotein that mediates cell-to-cell and cell-to-matrix interactions. It can bind fibrinogen, fibronectin, laminin, type $\mathrm{V}$ collagen and integrins $\alpha \mathrm{V} \beta 1, \alpha \mathrm{V} \beta 3$ and $\alpha \mathrm{IIb} \beta 3$, thus the significant reduction observed at $\mathrm{T} 1$ well agrees with its function. In fact, THBS-1 expression decreased at $\mathrm{T} 1$ for the inhibitory effect of clopidogrel loading dose on platelet reactivity, then after PCI it returned to be similar to T0 presumably because the plaque rupture and the vascular damage, induced by the stent deposition, may activate platelets and increase THBS-1 expression.

Besides, a class of proteins termed SNAREs (including SNAP-23, syntaxins) are involved in secretory granule/plasma membrane fusion events, crucial mechanisms for the amplification of platelet response. SNAP proteins are expressed on the extracellular surface of platelets [18] and specifically cleaved by calpain in activated cells [19]. From our results, the increased amount of the $\alpha$-SNAP at T1 may suggest that platelets, and particularly their exocytosis process, have been inhibited by clopidogrel loading dose.

Profilins play a central role in the regulation of de novo actin assembly by preventing spontaneous actin polymerization through the binding of actin monomers, and adding monomeric actin to the actinfilament ends $[20,21]$. Other cellular functions of profilin in membrane trafficking and lipid based signaling have also been reported. Phosphorylation of profilin resulted in a significant decrease in actin polymerization, indicating an increased affinity of phosphoprofilin toward actin [22]. Our results suggest a change of profilin-1 expression profile after clopidogrel administration with the appearance of additional spots at T1. The acidic products (spot $1=\mathrm{pI} 6.9$ and spot $2=$ pI 7.2) might be imputable to post-transductional modifications like phosphorylation or $\mathrm{N}$-acetylation. On the other hand serine phosphorylations N6-lysine (residues 103 and 106) and N-alanine (residue 2) acetylations have been documented for profilin-1 [23] and a direct correlation of these post-transductional modifications with the many functional effects of this protein has been reported [22,23].

In addition to these observations, the phosphorylation level of profilin and VASP appear to be modulated by clopidogrel. The 
inhibitory effect of clopidogrel on its platelet receptor P2Y12 is frequently evaluated through the intracellular marker VASP-P and it has been validated by clinical trials [24]. The interesting correlation between VASP and profilin suggests that platelet response, like the activation of surface receptors like integrin $\alpha \operatorname{IIb} \beta 3$ mediated by VASP and the cytoskeletal modification mediated by profilin, may be influenced by balanced phosphorylating reactions. Thus VASP phosphorylation and profilin dephosphorylation represents the inhibitory effect of clopidogrel on platelets.

Finally we observed that the expression of annexin- 5 was evident only at T1, after the clopidogrel loading dose. Annexin-5, a member of the calcium-binding proteins annexins expressed in human platelet, is a protein with potent and unique antithrombotic properties. The antithrombotic effect exerted by annexin- 5 seems to be mediated mainly by mechanical shielding of phospholipids, thereby reducing their availability for prothrombin binding and for the subsequent coagulation reactions. Behan et al. [25] demonstrated that the effect of clopidogrel results in an increased annexin- 5 binding, assessed by flow cytometry, thus a suppression of platelet pro-coagulant responses through annexin-5 and our results support their finding with the observation of an expression enhancement after clopidogrel treatment.

In addition to the cytoskeleton-related protein, we detected beta-arrestin 1 , member of the arrestin family, which participates to the P2Y receptors desensitization in platelets [26]. We interestingly observed its expression only at $\mathrm{T} 0$, indicating that the hyperactivation state of this ADP-dependent family of receptors became subsequently regulated by the clopidogrel therapy.

We finally observed some interesting changes of expressions in some groups of proteins, for example in those related to the energetic metabolism, like ubiquitin-like modifier-activating enzyme 1 , protein-L-isoaspartate-(D-aspartate) O-methyltransferase and nucleoside diphosphate kinase $\mathrm{B}$. This effect may be addressed to the rapid change of activating/inhibiting environment in which platelets were isolated. The increasing expression of STIP-1 over time may be ascribed to the endothelial damage provoked by the coronary angiography and revascularization procedure.

Current limitations of the proteomic approach concern the difficulty in the sample preparation in order to minimize the artifact, as in vitro activation, and avoid any cell contamination. Therefore we selected our platelet isolation method, using centrifugation and immediate freezing storage, in order to minimize the sample processing procedures and to maintain the platelets in their actual condition.

\section{Conclusion}

Platelet reactivity is a complex process regulated by multiple pathways and the interaction between those pathways and antiplatelet drugs represents one of the most debated topic of the last few years in cardiovascular research. We used a proteomic approach to reveal the physiologic mechanisms connected with the platelet hyper-activation and we found that the different expression of proteins associated with cytoskeleton (profilin-1, calpain, $\alpha$-SNAP and THBS-1), energetic metabolism (ubiquitin-like modifier-activating enzyme 1, protein-Lisoaspartate-(D-aspartate) O-methyltransferase and nucleoside diphosphate kinase B), oxidative stress (heat shock $70 \mathrm{kDa}$ protein 5 and anti-stress induced phosphoprotein 1) and inflammatory response may have a significant impact in this context.

\section{Authors' disclosures or potential conflicts of interest}

No authors declared any potential conflicts of interest.

\section{References}

[1] Kushner FG, Hand M, Smith Jr SC, King III SB, Anderson JL, Antman EM, et al. American College of Cardiology Foundation/American Heart Association Task Force on Practice Guidelines. 2009 Focused Updates: ACC/AHA Guidelines for the Management of Patients With ST-Elevation Myocardial Infarction (updating the 2004 Guideline and 2007 Focused Update) and ACC/AHA/SCAI Guidelines on Percutaneous Coronary Intervention (updating the 2005 Guideline and 2007 Focused Update): a report of the American College of Cardiology Foundation/American Heart Association Task Force on Practice Guidelines. Circulation 2009;120:2271-306.

[2] Steinhubl SR, Berger PB, Mann III JT, Fry ET, DeLago A, Wilmer C, et al. Early and sustained dual oral antiplatelet therapy following percutaneous coronary intervention: a randomized controlled trial. JAMA 2002;288:2411-20.

[3] Müller I, Seyfarth M, Rüdiger S, Wolf B, Pogatsa-Murray G, Schömig A, et al. Effect of a high loading dose of clopidogrel on platelet function in patients undergoing coronary stent placement. Heart 2001;85:92-3.

[4] Patti G, Colonna G, Pasceri V, Pepe LL, Montinaro A, Di Sciascio G. Randomized trial of high loading dose of clopidogrel for reduction of periprocedural myocardial infarction in patients undergoing coronary intervention: results from the ARMYDA-2 (Antiplatelet therapy for Reduction of MYocardial Damage during Angioplasty) study. Circulation 2005;111:2099-106.

[5] Michelson AD. Methods for the measurement of platelet function. Am J Cardiol 2009;103:20A-6A.

[6] Banfi C, Brioschi M, Marenzi G, De Metrio M, Camera M, Mussoni L, et al. Proteome of platelets in patients with coronary artery disease. Exp Hematol 2010;38: 341-50.

[7] Parguiña AF, Grigorian-Shamajian L, Agra RM, Teijeira-Fernández E, Rosa I, Alonso $\mathrm{J}$, et al. Proteins involved in platelet signaling are differentially regulated in acute coronary syndrome: a proteomic study. PLoS One 2010;5:e13404.

[8] Giusti L, Baldini C, Bazzichi L, Ciregia F, Tonazzini I, Mascia G, et al. Proteome analysis of whole saliva: a new tool for rheumatic diseases-the example of Sjögren's syndrome. Proteomics 2007;7:1634-43.

[9] Hochstrasser DF, Patchornik A, Merril CR. Development of polyacrylamide gels that improve the separation of proteins and their detection by silver staining. Anal Biochem 1988;173:412-23.

[10] Candiano G, Bruschi M, Musante L, Santucci L, Ghiggeri GM, Carnemolla B, et al. Blue silver: a very sensitive colloidal Coomassie G-250 staining for proteome analysis. Electrophoresis 2004;25:1327-33.

[11] Giusti L, Cetani F, Ciregia F, Da Valle Y, Donadio E, Giannaccini G, et al. A proteomic approach to study parathyroid glands. Mol Biosyst 2011;7:687-99.

[12] Keller A, Nesvizhskii AI, Kolker E, Aebersold R. Empirical statistical model to estimate the accuracy of peptide identifications made by MS/MS and database search. Anal Chem 2002;74:5383-92.

[13] Nesvizhskii AI, Keller A, Kolker E, Aebersold R. A statistical model for identifying proteins by tandem mass spectrometry. Anal Chem 2003;75:4646-58.

[14] Coppinger JA, Cagney G, Toomey S, Kislinger T, Belton O, McRedmond JP, et al. Characterization of the proteins released from activated platelets leads to localization of novel platelet proteins in human atherosclerotic lesions. Blood 2004;103:2096-104.

[15] Macaulay IC, Carr P, Gusnanto A, Ouwehand WH, Fitzgerald D, Watkins NA. Platelet genomics and proteomics in human health and disease. J Clin Invest 2005;115:3370-7.

[16] Croce K, Flaumenhaft R, Rivers M, Furie B, Furie BC, Herman IM, et al. Inhibition of calpain blocks platelet secretion, aggregation, and spreading. J Biol Chem 1999;274:36321-7.

[17] Schoenwaelder SM, Yuan Y, Jackson SP. Calpain regulation of integrin alpha IIb beta 3 signaling in human platelets. Platelets 2000;11:189-98.

[18] Flaumenhaft R, Rozenvayn N, Feng D, Dvorak AM. SNAP-23 and syntaxin-2 localize to the extracellular surface of the platelet plasma membrane. Blood 2007;110:1492-501.

[19] Rutledge TW, Whiteheart SW. SNAP-23 is a target for calpain cleavage in activated platelets. J Biol Chem 2002;277:37009-15.

[20] Jockusch BM, Murk K, Rothkegel M. The profile of profilins. Rev Physiol Biochem Pharmacol 2007:159:131-49.

[21] Witke W. The role of profilin complexes in cell motility and other cellular processes. Trends Cell Biol 2004;14:461-9.

[22] Sathish K, Padma B, Munugalavadla V, Bhargavi V, Radhika KV, Wasia R, et al. Phosphorylation of profilin regulates its interaction with actin and poly (Lproline). Cell Signal 2004;16:589-96.

[23] Ampe C, Markey F, Lindberg U, Vandekerckhove J. The primary structure of human platelet profilin: reinvestigation of the calf spleen profilin sequence. FEBS Lett 1988;228:17-21.

[24] Aleil B, Ravant C, Cazenave JP, Rochoux G, Heitz A, Gachet C. Flow cytometric analysis of intraplatelet VASP phosphorilation for the detection of clopidogrel resistance in patients with ischemic cardiovascular diseases. J Thromb Haemost 2005;3:85-92.

[25] Behan MW, Fox SC, Heptinstall S, Storey RF. Inhibitory effects of P2Y12 receptor antagonists on TRAP-induced platelet aggregation, procoagulant activity, microparticle formation and intracellular calcium responses in patients with acute coronary syndromes. Platelets 2005;2:73-80.

[26] Hoffmann C, Ziegler N, Reiner S, Krasel C, Lohse MJ. Agonist-selective, receptorspecific interaction of human P2Y receptors with beta-arrestin-1 and -2 . J Biol Chem 2008;283:30933-41 